\title{
Monotonicity of a profile of rankings with ties
}

\author{
Raúl Pérez-Fernández ${ }^{1}$, Irene Díaz ${ }^{2}$, Susana Montes $^{3}$, and Bernard De Baets ${ }^{1}$ \\ 1 KERMIT, Department of Data Analysis and Mathematical Modelling, \\ Ghent University, Coupure links 653, 9000 Gent, Belgium \\ \{raul. perezfernandez, bernard.debaets\} @ugent. be \\ 2 Department of Computer Science, University of Oviedo \\ sirene@uniovi.es \\ 3 Department of Statistics, O.R. and Mathematics Didactics, University of Oviedo \\ montes@uniovi.es
}

\begin{abstract}
A common problem in social choice theory concerns the aggregation of the rankings expressed by several voters. Two different settings are often discussed depending on whether the aggregate is assumed to be a latent true ranking that voters try to identify or a compromise ranking that (partially) satisfies most of the voters. In a previous work, we introduced the notion of monotonicity of a profile of rankings and used it for statistically testing the existence of this latent true ranking. In this paper, we consider different extensions of this property to the case in which voters provide rankings with ties.
\end{abstract}

Keywords: Social Choice; Monotonicity; Ranking; Signature.

\section{Introduction}

The aggregation of the rankings expressed by several voters is a classical problem in social choice theory that can be traced back to the 18-th century. Arrow [1] pointed out that "each individual has two orderings, one which governs him in his everyday actions, and one which would be relevant under some ideal conditions and which is in some sense truer than the first ordering. It is the latter which is considered relevant to social choice, and it is assumed that there is complete unanimity with regard to the truer individual ordering". From this reflection, one could conclude that there are two different settings for the aggregation of rankings: there exists a latent true ranking that voters try to identify, the goal of the aggregation being to identify said true ranking, or, contrarily, voters have conflicting opinions, the goal of the aggregation being to agree on a compromise ranking. In [2], we described a statistical test for testing the existence of a latent true ranking based on the notion of monotonicity of a profile of rankings.

Unfortunately, since the monotonicity of a profiles of rankings with ties has not been defined, the aforementioned statistical test cannot be used in case the 
rankings provided by the voters contain ties. This is a typical problem in real-life problems where voters might consider that two or more candidates are equally suitable [3]. Some existing methods for the aggregation of rankings, such as the method of Kemeny [4] and the method of Schulze [5], are explicitly defined to aggregate rankings with ties. Others, such as the Borda count [6], need to be adapted [7]. In the case of our statistical test, we will see that there is an immediate extension of the property of monotonicity, but we will also propose other extensions based on the notions of signature and ordered signature that might play an interesting role when using real-life data.

The remainder of the paper is structured as follows. In Section 2, we recall the notion of monotonicity of a profile of rankings. The natural generalization of this notion to rankings with ties is provided in Section 3. In Section 4, we introduce the notions of signature and ordered signature and discuss their relation with the property of monotonicity of a profile of rankings with ties. We end with some conclusions and open problems in Section 5.

\section{Monotonicity of a profile of rankings without ties}

We consider the problem where several voters express their preferences on a set $\mathscr{C}$ of $k$ candidates. In particular, each of the $r$ voters expresses a ranking $\succ_{j}$ on $\mathscr{C}$, i.e., the asymmetric part of a total order relation $\succeq_{j}$ on $\mathscr{C}$. The set of all possible rankings on $\mathscr{C}$ is denoted by $\mathcal{L}(\mathscr{C})$.

Each ranking $\succ$ on $\mathscr{C}$ defines an order relation $\Xi_{\succ}$ on $\mathcal{L}(\mathscr{C})$ according to how far two rankings in $\mathcal{L}(\mathscr{C})$ are from $\succ$ in terms of reversals ${ }^{4}$. For any $\succ_{i}, \succ_{j} \in \mathcal{L}(\mathscr{C})$, the fact that $\left(\succ_{i}, \succ_{j}\right) \in \underline{\Xi}_{\succ}$ is denoted by $\succ_{i} \unrhd_{\succ} \succ_{j}$.

Definition 1. Let $\mathscr{C}$ be a set of $k$ candidates and $\succ$ be a ranking on $\mathscr{C}$. The order relation $\unrhd_{\succ}$ on $\mathcal{L}(\mathscr{C})$ is defined as

$$
\unrhd_{\succ}=\left\{\left(\succ_{i}, \succ_{j}\right) \in \mathcal{L}(\mathscr{C})^{2} \mid \begin{array}{c}
\left(\forall\left(a_{i_{1}}, a_{i_{2}}\right) \in \mathscr{C}^{2}\right) \\
\left(\left(a_{i_{1}} \succ a_{i_{2}} \wedge a_{i_{1}} \succ_{j} a_{i_{2}}\right) \Rightarrow a_{i_{1}} \succ_{i} a_{i_{2}}\right)
\end{array}\right\} .
$$

Figure 1 displays the Hasse diagram of the order relation $\Xi_{\succ}$ on $\mathcal{L}(\mathscr{C})$ for the set of candidates $\mathscr{C}=\{a, b, c\}$ and the ranking $a \succ b \succ c$.

In [2], we described a statistical test for testing the existence of a latent true ranking. This test requires the given profile of rankings to be (close to being)

\footnotetext{
${ }^{4}$ A reversal is a switch of consecutive elements in a ranking. The minimum number of reversals needed for changing a given ranking into another one is measured by the Kendall distance function [8].
} 


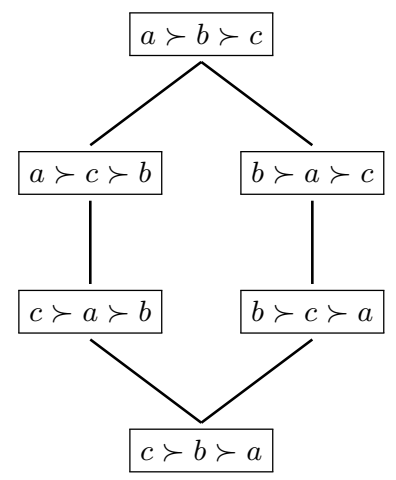

Fig. 1. Hasse diagram of the order relation $\unrhd_{\succ}$ on $\mathcal{L}(\mathscr{C})$ for the ranking $a \succ b \succ c$.

monotone, i.e., it requires the frequencies with which each ranking is expressed by the voters to be (close to being) decreasing on the Hasse diagram of the order relation $\Xi_{\succ_{0}}$, for some $\succ_{0} \in \mathcal{L}(\mathscr{C})$. This 'closeness to being monotone' was used for determining whether the hypothesis of existence of a latent true ranking should or should not be rejected. For more details, we refer to [2].

\section{Monotonicity of a profile of rankings with ties}

In real-life problems, voters might consider that two or more candidates are equally suitable, and, thus, express a tie between these candidates. In this case, every voter should be allowed to provide a weak order relation $\succsim_{j}$ on $\mathscr{C}$, i.e., a complete and transitive relation on $\mathscr{C}$ that might not be antisymmetric. Any weak order relation $\succsim$ can be written as the union of two relations $\succ$ and $\sim$, where $\succ$ (called a ranking with ties) represents the antisymmetric part of $\succsim$ and $\sim$ represents the symmetric part of $\succsim$. Note that $\sim$ is an equivalence relation, and, thus, partitions $\mathscr{C}$ into equivalence classes. The set of all rankings with ties on $\mathscr{C}$ is denoted by $\mathcal{L}^{*}(\mathscr{C})$. As a ranking is a particular case of a ranking with ties, it obviously holds that $\mathcal{L}(\mathscr{C}) \subseteq \mathcal{L}^{*}(\mathscr{C})$.

In case the voters express rankings with ties instead of rankings, the relation $\Xi_{\succ}$ needs to be extended to $\mathcal{L}^{*}(\mathscr{C})$. Note that, for a ranking with ties $\succ_{i}$, the conditions $a_{i_{1}} \succ_{i} a_{i_{2}}$ and $a_{i_{2}} \nsucc_{i} a_{i_{1}}$ are no longer equivalent. Therefore, the former unique condition for rankings (without ties) now needs to be divided in two parts. 
Proposition 1. Let $\mathscr{C}$ be a set of $k$ candidates and $\succ$ be a ranking on $\mathscr{C}$. The relation $\Xi_{\succ}$ defined $a s^{5}$

$$
\underline{\Xi}_{\succ}=\left\{\left(\succ_{i}, \succ_{j}\right) \in \mathcal{L}^{*}(\mathscr{C})^{2} \mid\left(\begin{array}{c}
\left(\forall\left(a_{i_{1}}, a_{i_{2}}\right) \in \mathscr{C}^{2}\right) \\
\left(a_{i_{1}} \succ a_{i_{2}} \wedge a_{i_{1}} \succ_{j} a_{i_{2}}\right) \Rightarrow\left(a_{i_{1}} \succ_{i} a_{i_{2}}\right) \\
\left(a_{i_{1}} \succ a_{i_{2}} \wedge a_{i_{2}} \succ_{i} a_{i_{1}}\right) \Rightarrow\left(a_{i_{2}} \succ_{j} a_{i_{1}}\right)
\end{array}\right)\right\}
$$

is an order relation on $\mathcal{L}^{*}(\mathscr{C})$.

Figure 2 displays the Hasse diagram of the order relation $\Xi_{\succ}$ on $\mathcal{L}^{*}(\mathscr{C})$ for the set of candidates $\mathscr{C}=\{a, b, c\}$ and the ranking $a \succ b \succ c$. Note that this Hasse diagram coincides with the one used by Kemeny [4] for defining a distance function on $\mathcal{L}^{*}(\mathscr{C})$.

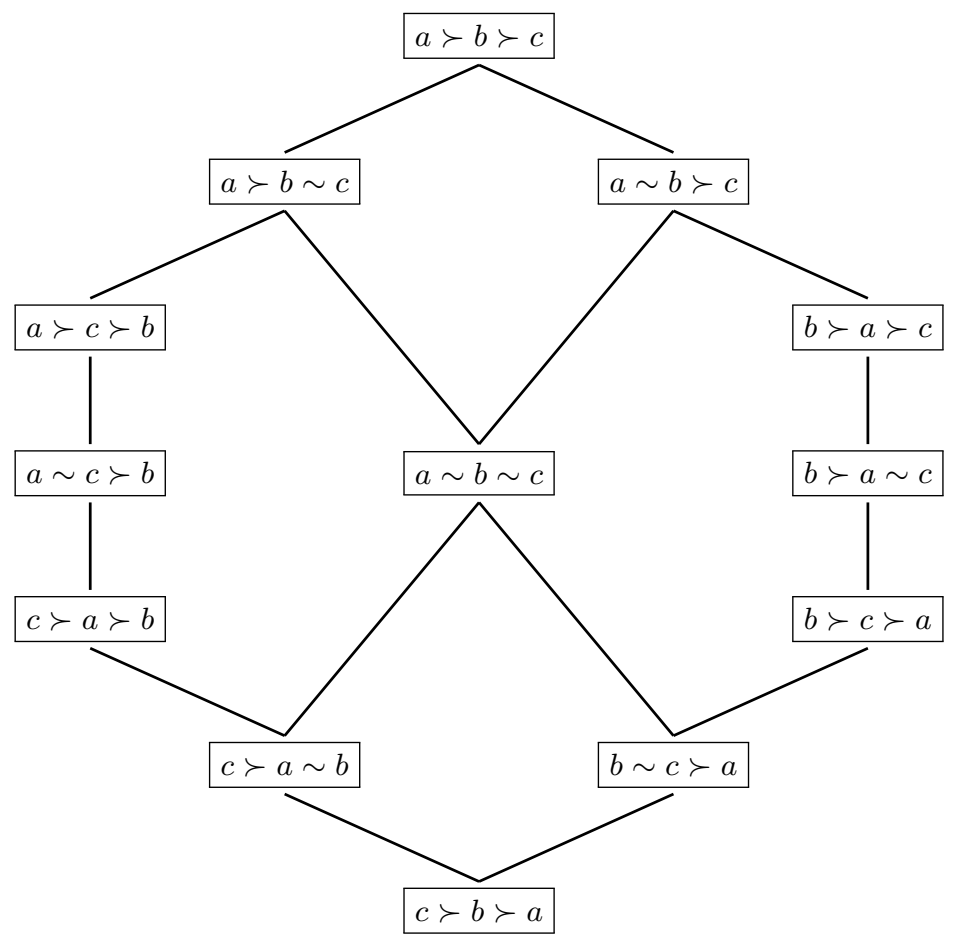

Fig. 2. Hasse diagram of the order relation $\Xi_{\succ}$ on $\mathcal{L}^{*}(\mathscr{C})$ for the ranking $a \succ b \succ c$.

\footnotetext{
${ }^{5}$ For any ranking $\succ$ on $\mathscr{C}$, the restriction of the relation $\unrhd_{\succ}$ on $\mathcal{L}^{*}(\mathscr{C})$ to $\mathcal{L}(\mathscr{C})$ coincides with the relation $\unrhd_{\succ}$ on $\mathcal{L}(\mathscr{C})$. Therefore, the use of the same notation is justified.
} 
The statistical test introduced in [2] might also be extended by requiring the frequencies with which each ranking with ties is expressed by the voters to be (close to being) decreasing on the Hasse diagram of the order relation $\unrhd_{\succ_{0}}$, for some $\succ_{0} \in \mathcal{L}(\mathscr{C})$.

Unfortunately, the inclination of voters towards expressing ties between candidates might prevent the frequencies from being decreasing on the Hasse diagram of the order relation $\unrhd_{\succ_{0}}$ (for any $\succ_{0} \in \mathcal{L}(\mathscr{C})$ ). For instance, the fact that the ranking with ties $a \sim b \sim c$ in Figure 2 would appear with the highest frequency in a given profile of rankings with ties will make the frequencies with which each ranking with ties is expressed to be far from being decreasing on the Hasse diagram of the order relation $\unrhd_{\succ_{0}}$, for any $\succ_{0} \in \mathcal{L}(\mathscr{C})$. Nevertheless, this might not be due to the absence of a true latent ranking, but due to the indecision of some of the voters. In particular, the frequencies with which the ranking with ties $a \sim b \sim c$ is expressed might be higher than that of $a \succ b \succ c$, even though the latter is the true latent ranking.

\section{Signatures and ordered signatures}

In this section, we study the notions of signature and ordered signature that will help to deal with the aforementioned problem.

Definition 2. Let $\mathscr{C}$ be a set of $k$ candidates.

(i) The signature $\mathscr{S}$ of a ranking with ties $\succ$ on $\mathscr{C}$, denoted by $\mathscr{S}(\succ)$, is a vector where the $i$-th component equals the size of the $i$-th equivalence class in $\succ$.

(ii) The ordered signature $\mathscr{O}$ of a ranking with ties $\succ$ on $\mathscr{C}$, denoted by $\mathscr{O}(\succ)$, is a vector where the $i$-th component equals the size of the $i$-th largest equivalence class in $\succ$.

The set of all the signatures on $\mathscr{C}$ is denoted by $\mathbb{S}(\mathscr{C})$ and the set of all the ordered signatures on $\mathscr{C}$ is denoted by $\mathbb{O}(\mathscr{C})$.

Remark 1. Each signature $\mathscr{S} \in \mathbb{S}(\mathscr{C})$ leads to a unique ordered signature $\mathscr{O} \in$ $\mathbb{O}(\mathscr{C})$ by ordering the numbers in $\mathscr{S}$ in a decreasing manner. Note that the lengths of $\mathscr{S}$ and $\mathscr{O}$ coincide. The fact that a signature $\mathscr{S}$ leads to an ordered signature $\mathscr{O}$ is denoted by $\mathscr{S} \rightsquigarrow \mathscr{O}$.

Example 1. Consider the set of candidates $\mathscr{C}=\{a, b, c\}$. The signature of the ranking with ties $a \succ b \succ c$ is the vector $(1,1,1)$ and its ordered signature is 
$(1,1,1)$. Therefore, it holds that

$$
\mathscr{S}(a \succ b \succ c)=\mathscr{O}(a \succ b \succ c)=(1,1,1) .
$$

Analogously, the signature of the ranking with ties $a \succ b \sim c$ is the vector $(1,2)$ and its ordered signature is $(2,1)$. Therefore, it holds that

$$
\mathscr{S}(a \succ b \sim c)=(1,2) \quad \text { and } \quad \mathscr{O}(a \succ b \sim c)=(2,1) .
$$

In general, the set of all signatures on $\mathscr{C}$ is given by:

$$
\mathbb{S}(\mathscr{C})=\{(1,1,1),(1,2),(2,1),(3)\} .
$$

Analogously, the set of all ordered signatures on $\mathscr{C}$ is given by:

$$
\mathbb{O}(\mathscr{C})=\{(1,1,1),(2,1),(3)\} .
$$

These (ordered) signatures can be used for defining two natural order relations on $\mathcal{L}^{*}(\mathscr{C})$. In the first order relation, only couples of rankings with ties belonging to $\unrhd_{\succ}$ and that have the same signature are considered to be comparable, while, in the second order relation, only couples of rankings with ties belonging to $\unrhd_{\succ}$ and that have the same ordered signature are considered to be comparable.

Proposition 2. Let $\mathscr{C}$ be a set of $k$ candidates and $\succ$ be a ranking on $\mathscr{C}$. The relation $\unrhd_{\succ}^{\mathbb{S}}$ defined as

$$
\underline{\bigotimes}_{\succ}^{\mathbb{S}}=\unrhd_{\succ} \cap\left\{\left(\succ_{i}, \succ_{j}\right) \in \mathcal{L}^{*}(\mathscr{C})^{2} \mid \mathscr{S}\left(\succ_{i}\right)=\mathscr{S}\left(\succ_{j}\right)\right\},
$$

is an order relation on $\mathcal{L}^{*}(\mathscr{C})$.

Proposition 3. Let $\mathscr{C}$ be a set of $k$ candidates and $\succ$ be a ranking on $\mathscr{C}$. The relation $\underline{\Xi}_{\succ}^{\mathbb{Q}}$ defined as

$$
\underline{\Xi}_{\succ}^{\mathbb{Q}}=\unrhd_{\succ} \cap\left\{\left(\succ_{i}, \succ_{j}\right) \in \mathcal{L}^{*}(\mathscr{C})^{2} \mid \mathscr{O}\left(\succ_{i}\right)=\mathscr{O}\left(\succ_{j}\right)\right\},
$$

is an order relation on $\mathcal{L}^{*}(\mathscr{C})$.

Figures 3 and 4 display the Hasse diagram of the order relations $\unrhd_{\succ}^{\mathbb{S}}$ and $\unrhd_{\succ}^{\mathbb{Q}}$ on $\mathcal{L}^{*}(\mathscr{C})$ for the set of candidates $\mathscr{C}=\{a, b, c\}$ and the ranking $a \succ b \succ c$. Note that we use dashed lines for separating sets of incomparable rankings with ties.

Obviously, there exists an immediate connection between the three relations.

Proposition 4. Let $\mathscr{C}$ be a set of $k$ candidates and $\succ$ be a ranking on $\mathscr{C}$. The following statement holds:

$$
\unrhd_{\succ}^{\mathbb{S}} \subseteq \unrhd_{\succ}^{\mathbb{Q}} \subseteq \unrhd_{\succ}
$$



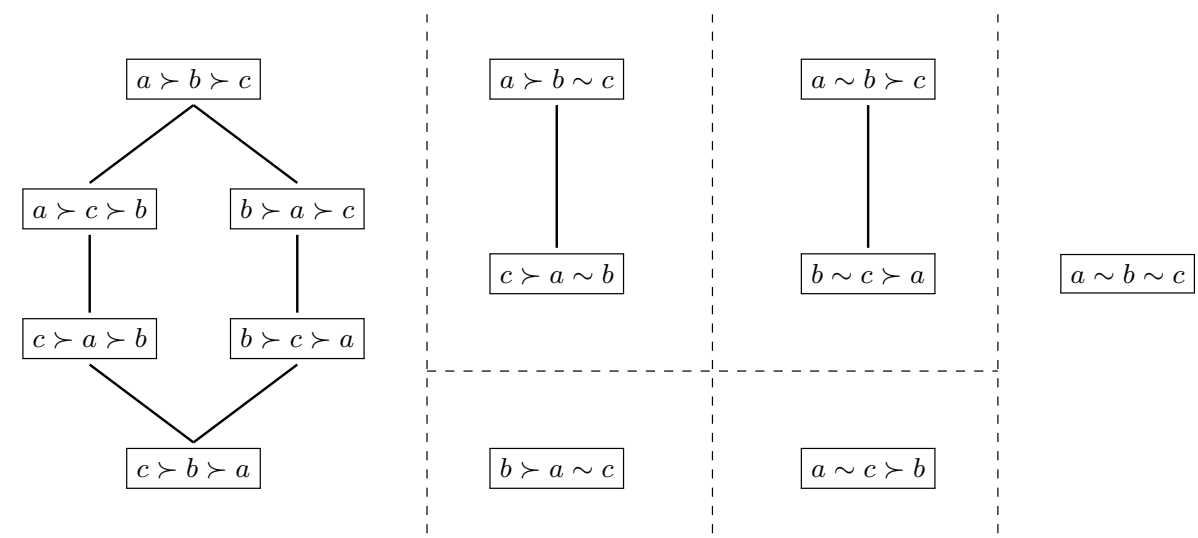

Fig. 3. Hasse diagram of the order relation $\unrhd_{\succ}^{\mathbb{S}}$ on $\mathcal{L}^{*}(\mathscr{C})$ for the ranking $a \succ b \succ c$.
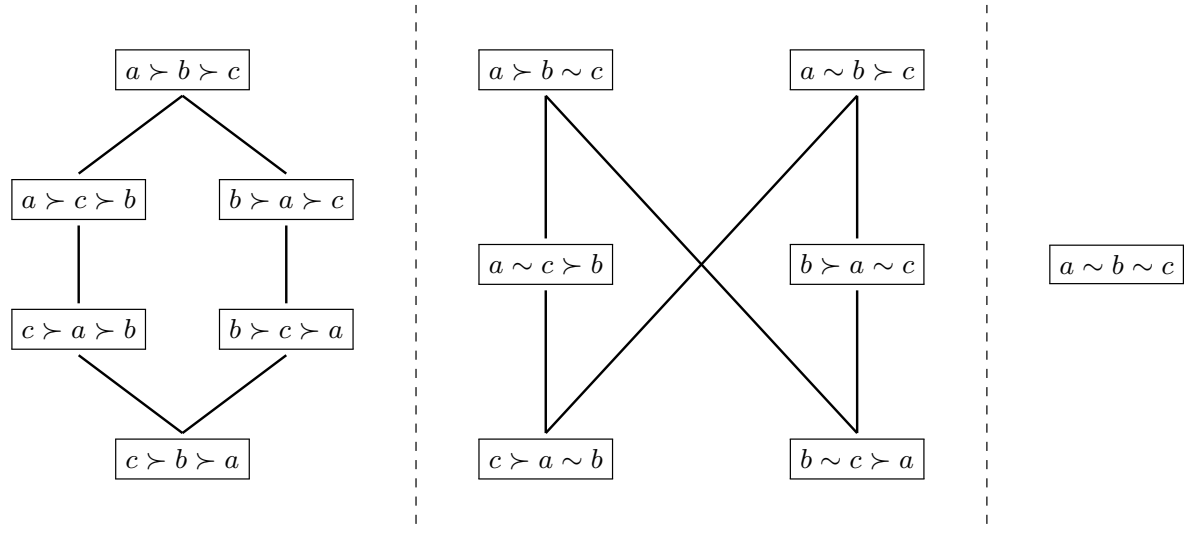

Fig. 4. Hasse diagram of the order relation $\unrhd_{\succ}^{\mathbb{Q}}$ on $\mathcal{L}^{*}(\mathscr{C})$ for the ranking $a \succ b \succ c$.

Consider the relation $\gg^{\mathbb{S}}$ on $\mathbb{S}(\mathscr{C})$, where ' $\mathscr{S}_{1} \gg^{\mathbb{S}} \mathscr{S}_{2}$ ' represents that the length of the signature $\mathscr{S}_{1}$ equals the length of the signature $\mathscr{S}_{2}$ plus one and, at the same time, the signature $\mathscr{S}_{2}$ can be obtained by merging two consecutive components of $\mathscr{S}_{1}$. For instance, the signature $(1,2)$ is obtained by merging the last two components of the signature $(1,1,1)$, therefore $(1,1,1) \gg^{\mathbb{S}}(1,2)$. We consider its pre-order closure ${ }^{6}$ for defining a natural order relation on $\mathbb{S}(\mathscr{C})$.

Proposition 5. Let $\mathscr{C}$ be a set of $k$ candidates. The relation $\geq^{\mathbb{S}}$, defined as the pre-order closure of $\gg^{\mathbb{S}}$, is an order relation on $\mathbb{S}(\mathscr{C})$.

${ }^{6}$ The pre-order closure of a relation $R$ is the smallest reflexive and transitive relation containing $R$ [9]. 
Analogously, a natural order relation can be defined for ordered signatures. Consider the relation $\gg^{\mathbb{O}}$ on $\mathbb{O}(\mathscr{C})$, where ' $\mathscr{O}_{1} \gg^{\mathbb{O}} \mathscr{O}_{2}$ ' represents that there exist two signatures $\mathscr{S}_{1}, \mathscr{S}_{2} \in \mathbb{S}(\mathscr{C})$ such that $\mathscr{S}_{1} \rightsquigarrow \mathscr{O}_{1}, \mathscr{S}_{2} \rightsquigarrow \mathscr{O}_{2}$ and $\mathscr{S}_{1} \gg^{\mathbb{S}}$ $\mathscr{S}_{2}$. For instance, for the ordered signatures $(2,1)$ and $(1,1,1)$, it holds that $(1,2) \rightsquigarrow(2,1),(1,1,1) \rightsquigarrow(1,1,1)$ and $(1,1,1) \gg^{\mathbb{S}}(1,2)$. Therefore, it holds that $(1,1,1) \gg^{\mathbb{Q}}(2,1)$. This relation is used for defining a natural order relation on $\mathbb{O}(\mathscr{C})$.

Proposition 6. Let $\mathscr{C}$ be a set of $k$ candidates. The relation $\geq^{\mathbb{Q}}$, defined as the pre-order closure of $\gg^{\mathbb{O}}$, is an order relation on $\mathbb{O}(\mathscr{C})$.

Figures 5 and 6 display the Hasse diagram of the order relations $\geq^{\mathbb{S}}$ and $\geq \mathbb{0}$ on $\mathbb{S}(\mathscr{C})$ for a set $\mathscr{C}$ of three and of four candidates.
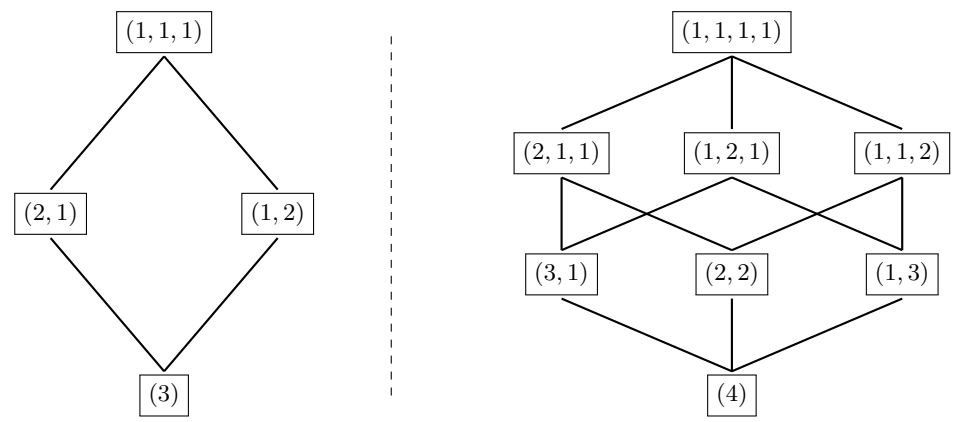

Fig. 5. Hasse diagram of the order relation $\geq^{\mathbb{S}}$ on $\mathbb{S}(\mathscr{C})$ for a set $\mathscr{C}$ of three (left) and of four (right) candidates.

These order relations on the set of (ordered) signatures can be used for defining four natural order relations on $\mathcal{L}^{*}(\mathscr{C})$, where only couples of rankings with ties belonging to $\unrhd_{\succ}$ and satisfying these additional requirements are considered comparable elements.

Proposition 7. Let $\mathscr{C}$ be a set of $k$ candidates and $\succ$ be a ranking on $\mathscr{C}$. The relations defined as

$$
\begin{aligned}
& \underline{\succ}_{\succ}^{\mathbb{S}_{\downarrow}}=\unrhd_{\succ} \cap\left\{\left(\succ_{i}, \succ_{j}\right) \in \mathcal{L}^{*}(\mathscr{C})^{2} \mid \mathscr{S}\left(\succ_{i}\right) \geq^{\mathbb{S}} \mathscr{S}\left(\succ_{j}\right)\right\}, \\
& \underline{\unlhd}_{\succ}^{\mathbb{S} \uparrow}=\underline{\Xi}_{\succ} \cap\left\{\left(\succ_{i}, \succ_{j}\right) \in \mathcal{L}^{*}(\mathscr{C})^{2} \mid \mathscr{S}\left(\succ_{j}\right) \geq^{\mathbb{S}} \mathscr{S}\left(\succ_{i}\right)\right\}, \\
& \underline{\succ}_{\succ}^{\mathbb{O}_{\downarrow}}=\unrhd_{\succ} \cap\left\{\left(\succ_{i}, \succ_{j}\right) \in \mathcal{L}^{*}(\mathscr{C})^{2} \mid \mathscr{O}\left(\succ_{i}\right) \geq^{\mathbb{O}} \mathscr{O}\left(\succ_{j}\right)\right\}, \\
& \underline{\Xi}_{\succ}^{\mathbb{O}_{\uparrow}}=\underline{\Xi}_{\succ} \cap\left\{\left(\succ_{i}, \succ_{j}\right) \in \mathcal{L}^{*}(\mathscr{C})^{2} \mid \mathscr{O}\left(\succ_{j}\right) \geq^{\mathbb{O}} \mathscr{O}\left(\succ_{i}\right)\right\},
\end{aligned}
$$

are four order relations on $\mathcal{L}^{*}(\mathscr{C})$. 

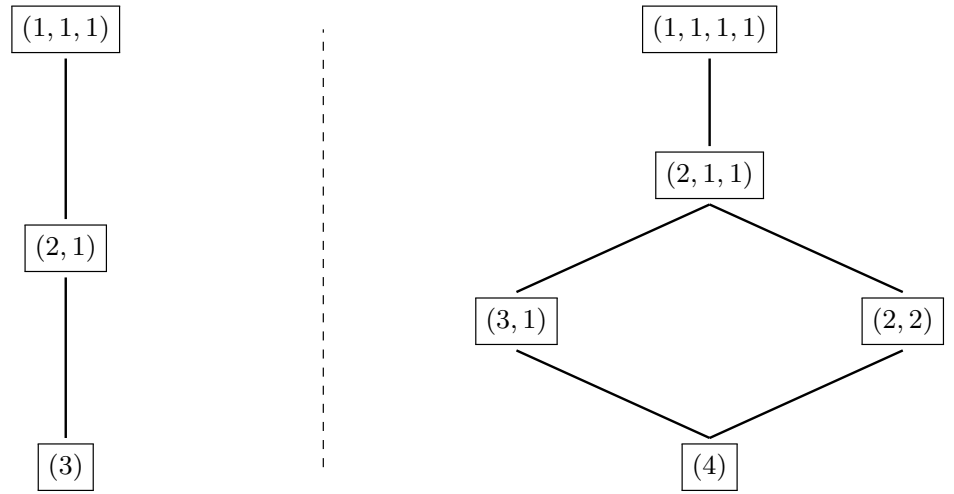

Fig. 6. Hasse diagram of the order relation $\geq^{\mathbb{O}}$ on $\mathbb{O}(\mathscr{C})$ for a set $\mathscr{C}$ of three (left) and of four (right) candidates.

For instance, we illustrate in Figure 7 the Hasse diagram of the order relation $\unrhd_{\succ}^{\mathbb{O}_{\downarrow}}$ on $\mathcal{L}^{*}(\mathscr{C})$ for the set of candidates $\mathscr{C}=\{a, b, c\}$ and the ranking $a \succ b \succ c$. The fact that the frequencies of the rankings with ties are decreasing on the Hasse diagram of $\unrhd_{\succ}^{\mathbb{O}_{\downarrow}}$ (and not on that of $\unrhd_{\succ}$ ) would imply that voters do not express ties between candidates as often as they express strict preferences.

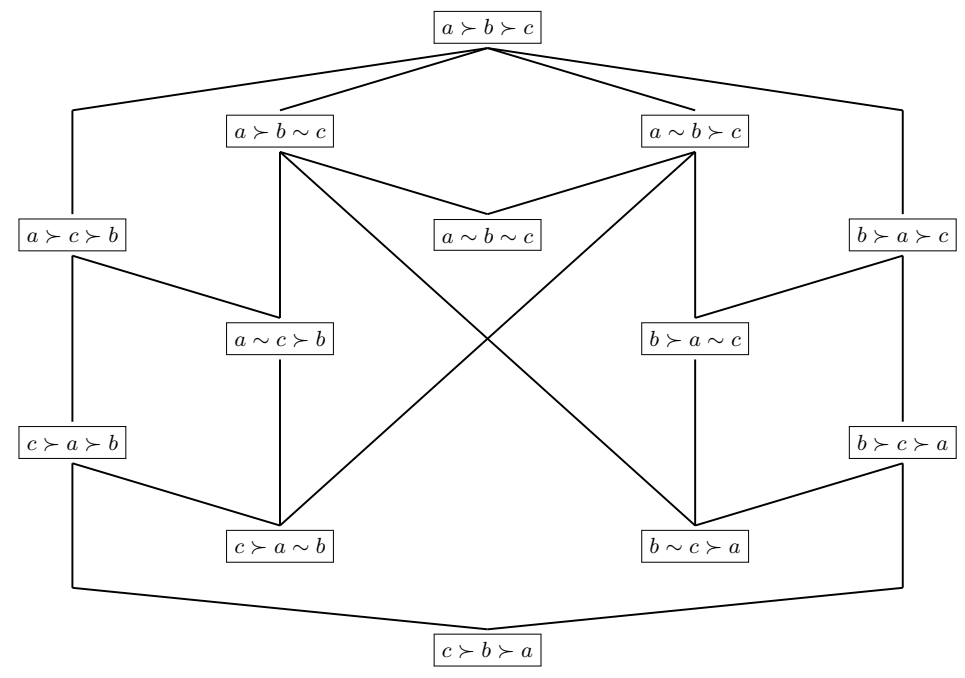

Fig. 7. Hasse diagram of the order relation $\unrhd_{\succ}^{\mathbb{O}_{\downarrow}}$ on $\mathcal{L}^{*}(\mathscr{C})$ for the ranking $a \succ b \succ c$. 
All different relations between the seven order relations are described in Figure 8 . One can see that $\unrhd_{\succ}$ contains the other six order relations, whereas $\unrhd_{\succ}^{\mathbb{S}}$ is contained in the other six order relations.

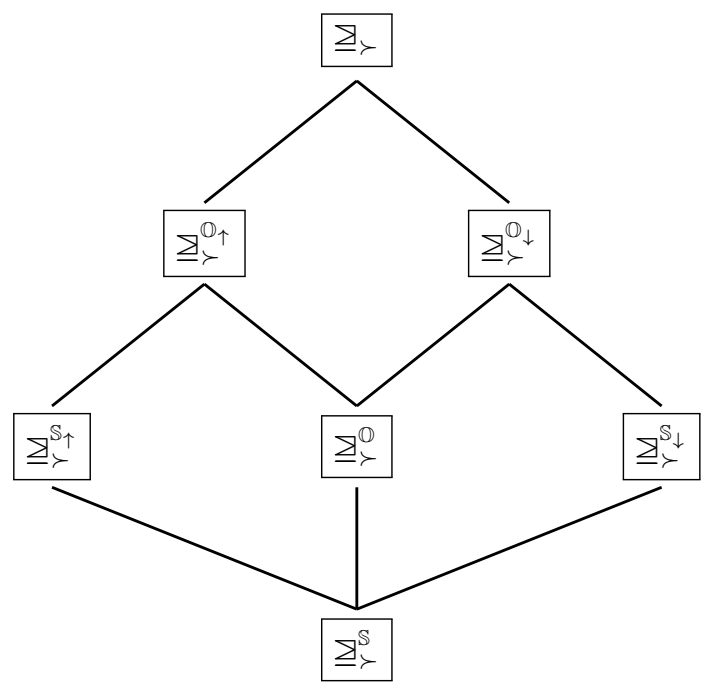

Fig. 8. Hasse diagram of the order relation $\subseteq$ on the set of all order relations on $\mathcal{L}^{*}(\mathscr{C})$ defined in this section.

The statistical test introduced in [2] might now be extended by requiring the frequencies with which each ranking is expressed to be (close to being) decreasing on the Hasse diagram of one of the seven different order relations. Obviously, since $\unrhd_{\succ}^{\mathbb{S}}$ is contained in the other six order relations, decreasingness w.r.t. $\unrhd_{\succ}^{\mathbb{S}}$ will be the closest to being satisfied, and, thus, the most unlikely of leading to a rejection of the hypothesis of existence of a latent true ranking. Different conclusions concerning the tendency of voters towards expressing ties can be drawn from the results of the test by selecting different order relations among the seven ones defined in this manuscript.

\section{Conclusions and open problems}

In this paper, we have generalized the notion of monotonicity of a profile of rankings to rankings with ties. In the future, we will aim at adapting a previouslyproposed statistical test for the existence of a true latent ranking based on the property of monotonicity of a profile of rankings to the setting in which rankings with ties are provided. We have conjectured that, in real-life data, the 
inclination of voters towards expressing ties between candidates might play a big role in the rejection of the test, and, thus, we have proposed six alternative definitions of monotonicity of a profile of rankings with ties based on the notions of signature and ordered signature. A thorough study on the influence of the chosen notion of monotonicity (and especially that of the least restrictive one $\unlhd_{\succ}^{\mathbb{S}}$ ) in the statistical test is yet to be addressed. Moreover, in this paper, we have restricted the results of the aggregation of the rankings with ties given by the voters to be a ranking without ties. The case in which the result of this aggregation is also allowed to be a ranking with ties will be analysed in the near future.

\section{Acknowledgments}

This research has been partially supported by the Research Foundation of Flanders (FWO17/PDO/160) and by the Ministry of Economy, Industry and Competitiveness (TIN2017-87600-P).

\section{References}

1. Arrow, K.J.: Social Choice and Individual Values. Wiley, New York (1963)

2. Pérez-Fernández, R., Alonso, P., Díaz, I., Montes, S., De Baets, B.: Monotonicity as a tool for differentiating between truth and optimality in the aggregation of rankings. Journal of Mathematical Psychology 77 (2017) 1-9

3. Pérez-Fernández, R., Alonso, P., Díaz, I., Montes, S., De Baets, B.: Monotonicitybased consensus states for the monometric rationalisation of ranking rules and how they are affected by ties. International Journal of Approximate Reasonig 91 (2017) $131-151$

4. Kemeny, J.G.: Mathematics without numbers. Daedalus 88(4) (1959) 577-591

5. Schulze, M.: A new monotonic, clone-independent, reversal symmetric, and Condorcet-consistent single-winner election method. Social Choice and Welfare 36 (2011) 267-303

6. Borda, J.C.: Mémoire sur les Élections au Scrutin. Histoire de l'Académie Royale des Sciences, Paris (1781)

7. Black, D.: Partial justification of the Borda count. Public Choice 28 (1976) 1-15

8. Kendall, M.G.: A new measure of rank correlation. Biometrika 30 (1938) 81-93

9. Foldes, S.: Fundamental Structures of Algebra and Discrete Mathematics. Wiley, New York (1994) 